\title{
A wind tunnel study of organised and turbulent air motions in urban street canyons
}

\author{
P. Kastner-Klein ${ }^{\mathrm{a}}$, E. Fedorovich ${ }^{\mathrm{b}, *}$, M.W. Rotach ${ }^{\mathrm{a}}$ \\ ${ }^{a}$ Institute for Climate Research, Swiss Federal Institute of Technology (ETH), Zürich 8057, Switzerland \\ ${ }^{\mathrm{b}}$ School of Meteorology, University of Oklahoma, Energy Center, 100 East Boyd, \\ Norman, OK 73019-1013, USA
}

\begin{abstract}
High concentrations of car-exhaust gases in urban street canyons are typically associated with low wind velocities or situations when the wind blows perpendicular to the canyon axis. The latter flow configuration has been studied in a wind tunnel model of a street canyon. The mean flow and turbulence structure have both been investigated and comparisons have been carried out with results of full-scale flow measurements in urban street canyons. A qualitative similarity has been found between the results of atmospheric measurements and flow characteristics in the modelled street canyon. Data from all employed sources give evidence of a flow acceleration (in some cases, rather sharp) above roof level. Additionally, the effects of traffic on the organised and turbulent components of airflow in the canyon have been quantified. The experimental data show significant differences in flow and turbulence patterns corresponding to the model cases of one-way and two-way traffic. (C) 2001 Elsevier Science Ltd. All rights reserved.
\end{abstract}

Keywords: Wind; Turbulence; Dispersion; Traffic; Wind tunnel modelling; Field data

\section{Introduction}

Street canyons of big cities are usually rather poorly ventilated by natural air motions related to the external wind. Even when the wind is strong, the cloud of exhaust gases produced by traffic can be trapped in the lower portion of the canyon. It has been shown numerically in [1-3], and by means of wind tunnel modelling, see [3-5], that flow and dispersion patterns inside the canyon essentially depend on the

\footnotetext{
*Corresponding author. Tel.: + 1-405-325-1197; fax: + 1-405-325-7689.

E-mail address: fedorovich@ou.edu (E. Fedorovich).
} 
canyon geometry, specified in the first approximation by the canyon depth-to-width and length-to-depth ratios, and by the building and roof shapes. Particularly unfavourable ventilation conditions have been identified in situations when the wind is oriented perpendicular to the canyon axis and the depth-to-width (aspect) ratio of the canyon is large, of the order of one or more. Usually, such situations are associated with a weak organised in-canyon air circulation that has a form of a vortex (or several vortices) with axe(s) directed along the canyon. This vortex-like air motion is unsteady and intermittent. In many instances, it poorly interacts with the external flow above the canyon and does not significantly contribute to the removal of exhaust gases from the canyon.

Another component of air motion in the canyon is the turbulent one. Its role in the redistribution of pollutant concentration inside the canyon can be rather important. It is not clear, however, whether the turbulent diffusion could effectively wash the pollutant material out of the canyon. Very few full-scale studies, as well as wind tunnel experiments have been performed so far where organised and turbulent components of three-dimensional flow in the canyon have been measured with the spatial resolution sufficient for a quantitative description of the flow pattern in the canyon.

In the present study, wind tunnel measurements of mean flow and turbulent fluctuations in an idealised street canyon have been performed. The aspect ratio of the model street canyon, formed of two parallel building rows, has been equal to one. The details of the experimental setup and measurement technique employed in the wind tunnel experiments are given in Section 2 of the paper. The measurement data have been compared with available data from full-scale experiments with similar flow configurations.

Organised and turbulent components of airflow measured in the canyon and interaction between them will be considered in Section 3. In that section, a mechanism of turbulent exchange across the top of the canyon will also be discussed and the problem of choosing representative scales for mean flow and turbulence characteristics inside and above the canyon will be addressed.

Another topic of the present study is the effect of city traffic on the airflow inside the street canyon. It is still an open question whether moving cars, which pollute air in the canyon, can at the same time produce air motions strong enough to contribute to the ventilation of the canyon. There are very few available full-scale measurement data on local air motions generated by motor vehicles in urban street canyons. The most frequently used tools for estimating vehicle-induced effects on pollutant transport in the urban environment are numerical models. In these models, rather arbitrary assumptions are usually drawn to parameterise the impact of vehicles on the in-canyon flow. Existing parameterisation schemes typically do not distinguish between traffic-generated air motions of different nature and scale. Some models, (among them, for instance, those of Hertel and Berkowicz [6], Sini et al. [2], and Stern and Yamartino [7]), merely consider the whole spectrum of these motions as traffic-induced turbulence. However, experimental studies of Eskeridge and Hunt [8], and Delaunay and Houseaux [9] provide a certain evidence of semi-organised, advection-type air motions in a street with moving vehicles. 
In Section 4 of the paper we show and discuss results from a series of wind tunnel experiments aimed at the evaluation of the traffic contribution to air motions in the model street canyon. The traffic has been simulated using an experimental approach described in Refs. [10,12]. The cases of both one-way and two-way traffic have been studied. As the reference case, the flow field in the canyon without traffic has been employed. First, the examples of concentration distributions in the canyon with different traffic situations will be shown. In the further analysis, we will focus on the specific features of mean flow and turbulence fields corresponding to these situations.

The summary of experimental results and conclusions are presented in Section 5.

\section{Experimental setup and measurement technique}

The experiments have been performed in the atmospheric boundary layer wind tunnel of the University of Karlsruhe (UniKa), Germany. A description of this facility is given in Refs. [11,12]. In the wind tunnel, a scaled analogue of the atmospheric neutrally stratified boundary-layer flow is reproduced. This is achieved with the aid of vortex generators installed at the entrance of the test section and by means of roughness elements mounted on the wind tunnel floor. The typical boundary-layer depth in the vicinity of the street-canyon location is of the order of $50 \mathrm{~cm}$. In the present study, the height of the buildings forming the canyon has been $12 \mathrm{~cm}$, and their length has been either $60 \mathrm{~cm}$ or $120 \mathrm{~cm}$. The distance between the buildings has been chosen to be $12 \mathrm{~cm}$. This corresponds to the canyon aspect ratio equal to 1 and to the length-to-depth ratios equal to 5 (short canyon) or 10 (long canyon). In all experimental configurations to be considered in this paper, the external wind flow has been directed perpendicular to the axis of the canyon, see Fig. 1, and the $X$ axis has been oriented along the direction of external wind.

Rectangular plates mounted on two moving belts stretched along the canyon simulate the two-lane traffic in an urban street. This approach towards the simulation of traffic in wind tunnel models of urban airflow was first proposed in Ref. [13]. Its application in the UniKa wind tunnel is described in Ref. [10]. To enable the reproduction of situations with one-way or two-way traffic, the belts can be moved in alternative directions with prescribed velocity.

Mean flow and turbulence measurements in the tunnel were conducted with a laser Doppler velocimeter. In most of measurement locations inside the canyon and above it, the components of the velocity vector across the canyon ( $u$, the $x$-component of velocity) and along the canyon ( $v$, the $y$-component of velocity) were measured. In several locations, also $w$, the $z$-component of velocity, was registered. From the obtained time series, mean flow parameters and one-point, second-order turbulence statistics have been derived.

In parallel to the flow parameters, mean concentration values of a passive tracer gas have been measured at the building walls. The tracer gas has been emitted from two line sources co-located with the traffic lanes. The concentration patterns have been analysed in conjunction with the mean flow and turbulence fields. 


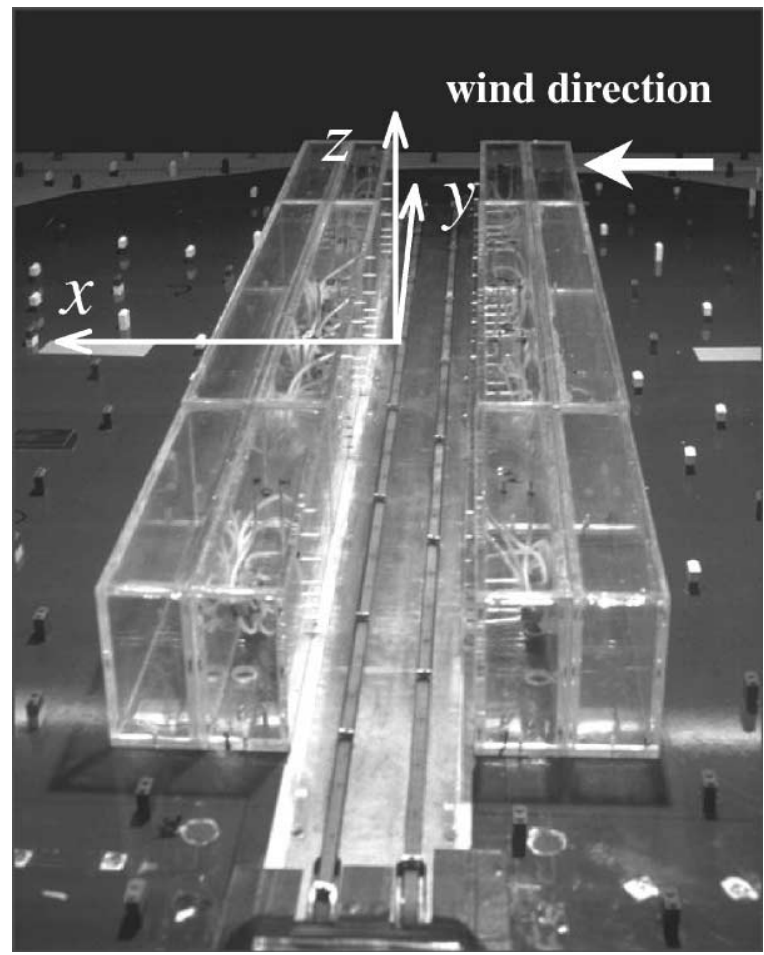

Fig. 1. Model of a street canyon in the UniKa atmospheric boundary layer wind tunnel.

\section{Mean flow and turbulence characteristics in the canyon without traffic}

The first group of experimental results to be presented includes measurement data on mean flow and turbulence in the case without traffic. The wind tunnel data will be analysed in comparison with results of corresponding full-scale measurements in urban street canyons by Rotach [14] and Louka [15].

A comparison of profiles of the modulus of the longitudinal mean flow component, $|u|$, is given in Fig. 2a. The rms values of the across-the-canyon $\left(\sigma_{u}\right)$ and along-the-canyon $\left(\sigma_{v}\right)$ velocity fluctuations are compared in Figs. $2 \mathrm{~b}$ and c, respectively.

The vertical coordinate is scaled with the building height $H$, and the measurement data are normalised by $u(H)$, the value of the mean wind speed at $z=H$. Such choice for the normalisation parameter was primarily determined by the availability of $u(H)$ for all compared datasets. Nevertheless, as will be shown below, this normalisation cannot be considered optimal for presenting velocity data from different wind tunnel and field experiments.

The wind tunnel data in Fig. 2 refer to the central (XoZ), plane of the canyon, and represent averaged values from seven vertical profiles inside the canyon (one of them 
(a)

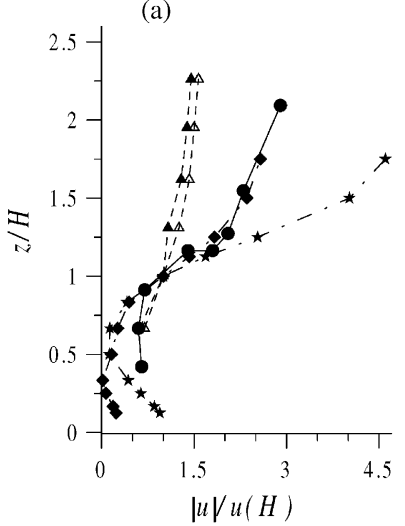

(b)

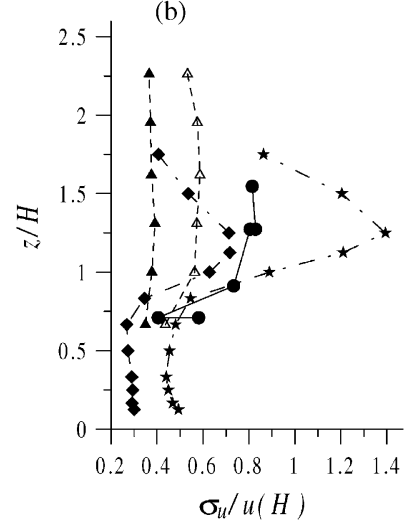

(c)

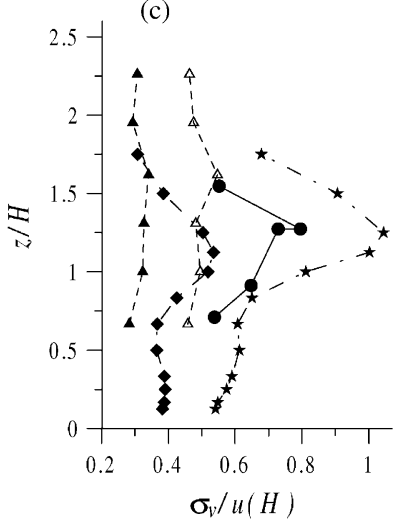

Fig. 2. Normalised profiles of the modulus of the longitudinal mean flow velocity component (a) and rms values of longitudinal (b) and lateral velocity (c) fluctuations inside and above an urban street canyon. The wind tunnel measurement data are given by stars for the $120 \mathrm{~cm}$-long canyon and by diamonds for the $60 \mathrm{~cm}$-long canyon; the urban data from Rotach [14] are presented by filled circles; and the data from "smooth-rough" and "rough-rough" cases of Louka [15] are shown by filled and open triangles, respectively.

in the centre of the canyon, and the rest located at equal distance intervals from the centre to the walls). In the experiments of Louka [15], the "smooth-rough" case corresponded to the building arrangement without any large-scale obstructions affecting the approach flow, and in the "rough-rough" case several additional buildings were located upwind the canyon.

The mean flow profiles from different datasets shown in Fig. 2a considerably diverge inside the canyon as well as above it. The closest conformity of the wind tunnel data with field measurements is observed in profiles from the $60 \mathrm{~cm}$-long wind tunnel canyon and from the full-scale study of Rotach [14]. The difference between the velocity data for the $120 \mathrm{~cm}$ - and $60 \mathrm{~cm}$-long wind tunnel canyons is mainly due to a rather small velocity value $u(H)$ in the case of the long canyon. Our wind tunnel experiments indicate that the mean-velocity field in the central plane of the long canyon is not affected by the lateral (with respect to the external flow) exchange of momentum, whilst in the case of the short canyon the momentum is rather effectively transported from the outlets of the canyon to its central plane. This leads to larger velocity values at the building-height level in the central plane of the short canyon.

One may suppose that the lateral exchange of momentum (also associated with influence of street crossings on the velocity field, see Ref. [11]) can play a significant role in real urban street canyons. Such lateral exchange is also a probable reason for the comparatively weak vortex-like circulation within the short canyon. This circulation is stronger in the long wind tunnel canyon and in the full-scale urban canyon from the study of Rotach [14]. Note that in the wind tunnel experiments the actual velocity values in the lower portion of the canyon were negative. During the field measurements the sign of mean velocity inside the canyon was not defined. 
The vertical distributions of velocity in the experiments of Louka [15] appear to be rather uniform with height as compared with the data of Rotach [14] and with the wind tunnel results. Probable reasons for this behaviour of velocity profiles from [15] may be the smaller canyon aspect ratio (0.7), slightly slanted roofs of the buildings, and deviations of the external flow from the direction perpendicular to the canyon axis.

The rms values of $u$ and $v$ velocity components from the wind tunnel and field experiments (Figs. 2b and c) are rather scattered as well. This scatter, like in the case of mean flow velocity profiles, is mainly caused by the divergence of the $u(H)$ values used for the normalisation of statistics. It gives grounds to assume that the velocity of the undisturbed flow at some sufficiently large elevation above the canyon could be more appropriate for the normalisation of the measured mean flow and rms velocity values inside and above the canyon. Unfortunately, the free stream velocity was not determined in the full-scale experiments, from which the data are used here for comparison.

It is possible to notice, however, that the features of the rms vertical distributions of both velocity components in Figs. $2 b$ and $c$ are quite similar for the data originating from one and the same source. The wind tunnel $u$ and $v$ rms values are nearly constant with height in the lower portion of the canyon. They reach their maxima slightly above the roof level. The field data of Rotach [14] display a similar tendency in this region of the flow. Much less pronounced maxima are observed in the profiles measured by Louka [15]. They show rather small variation of turbulence intensity with height above the canyon and the rms velocity values in the "rough-rough" case are persistently larger than in the "smooth-rough" case.

It should be mentioned on this occasion that the experimental setup employed in the wind tunnel study corresponds to a rather idealized case of an isolated canyon formed by two parallel buildings. Recent wind tunnel experiments of Brown et al. [16] with an array of such canyons have shown that the enhancement of turbulent fluctuations at the roof level is typically pronounced in the first few canyons from the array. In the subsequent canyons located downwind, more homogeneous vertical distributions of velocity variances have been observed.

While comparing the wind tunnel measurement and field observation data on flow parameters in urban street canyons one should also keep in mind that they are obtained under very different experimental conditions. Controlled approach flow conditions in the wind tunnel and simplified geometry of the model street canyon provide measured flow fields with strong gradients. In nature, on the other hand, the large-scale flow disturbances caused by the variation of wind direction and/or effects of hydrostatic stability tend to smooth the flow irregularities and lead to a smaller spatial variability of measured mean flow and turbulence fields. In the laboratory, data for one flow case are usually obtained with a fixed experimental setup, whilst the analogous flow case in the field can be studied in a sequence of separate experiments and corresponding data are collected over specific days as in the case study of Louka [15] or averaged over events with particular meteorological conditions as in the study of Rotach [14]. 
(a) $u / u(4 H)$

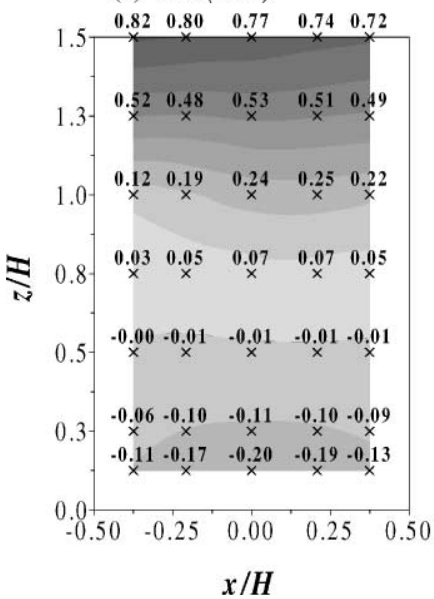

(b) $\sigma_{u} / u(4 H)$

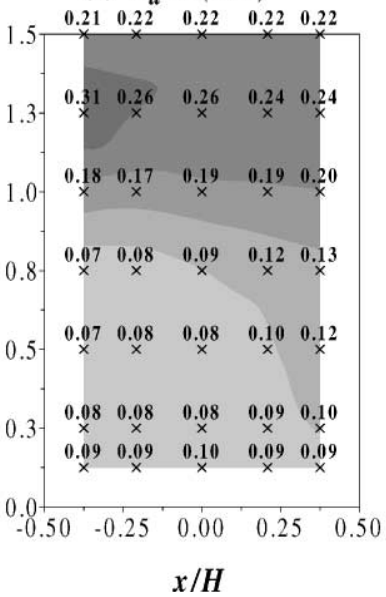

(c) $\sigma_{v} / u(4 H)$

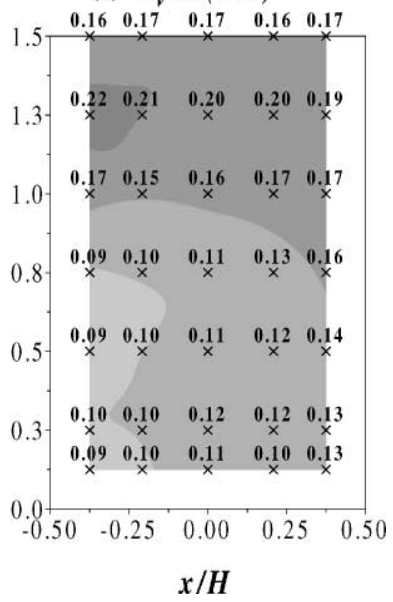

Fig. 3. Normalised distributions of the longitudinal mean flow velocity component (a), and rms values of longitudinal (b) and lateral velocity (c) fluctuations measured in the wind tunnel inside and above the $120 \mathrm{~cm}$-long street canyon without traffic. See text for details.

Details of the mean and rms velocity distributions inside and above an urban street canyon can be seen in Fig. 3, where the results of measurements performed in the central plane of the wind tunnel model of the long $(120 \mathrm{~cm})$ canyon are presented. The data are normalised by the wind velocity of the external flow at $z=4 H$ above the floor of the tunnel. In the pattern of mean flow velocity (Fig. 3a), one may clearly see the re-circulation zone (the in-canyon vortex) with almost zero velocity values in its centre. The near-surface negative mean air motion associated with such vortexlike motion effectively transports the emitted tracer towards the leeward wall of the canyon. This results in enhanced concentration values near the wall at the roadside level (see concentration distribution at the wall in Fig. 4). Immediately above the roof level $(z / H=1)$, a region of strong mean flow shear is located with sharp vertical gradients of the mean velocity.

The intensity of velocity fluctuations (Figs. $3 b$ and c) is rather uniformly distributed in the canyon without traffic. The fluctuations of both velocity components have nearly the same magnitude, which suggests the approximate isotropy of horizontal velocity fluctuations in the centre of the canyon. A slight increase of rms velocity values takes place immediately above the canyon, where the turbulence production is intensified due to the flow shear that was identified in the mean flow pattern. It is possible to assume that this shear can produce a certain sheltering effect on the vertical turbulent transport of admixture across the canyon top and thus hampers the ventilation of the canyon. Fedorovich and Kaiser [17] have observed a similar turbulence-blocking effect in the case of shear sheltering of buoyancy-generated turbulence. The related physical analysis is presented in Ref. [18]. 


\section{reference case: no moving vehicles}

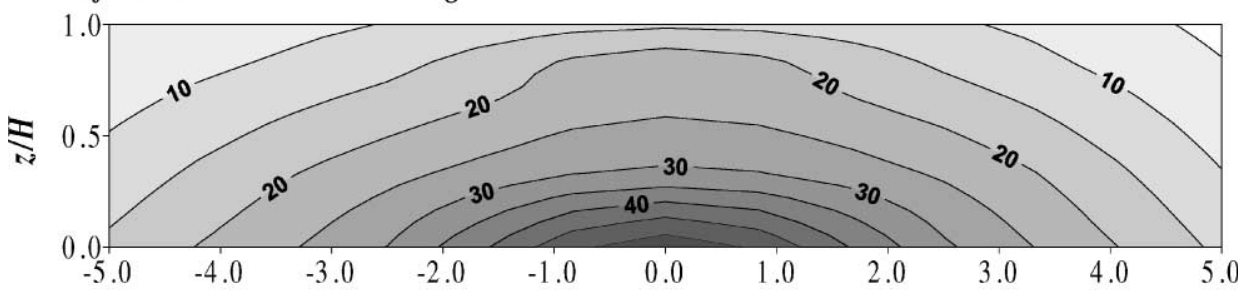

two-way traffic: vehicles moving with $v=30 \mathrm{~km} / \mathrm{h}$

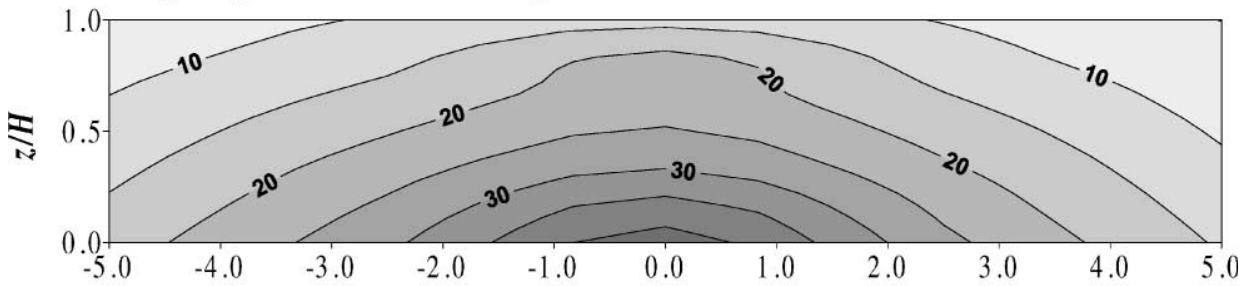

one-way traffic: vehicles moving with $v=30 \mathrm{~km} / \mathrm{h}$

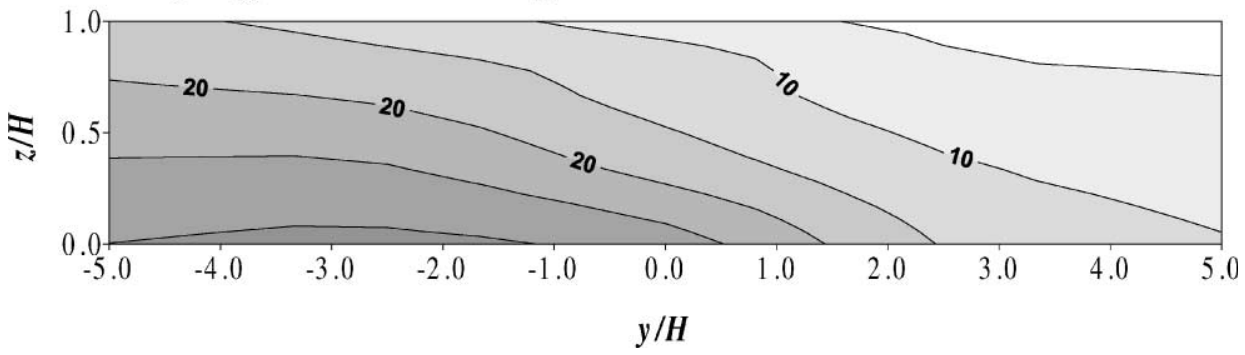

Fig. 4. Distributions of normalised concentration $c_{*}=c U H L_{\mathrm{s}} / E_{\mathrm{s}}(c$ is the measured concentration, $U$ is the reference wind velocity, $H$ is the canyon depth, $L_{\mathrm{s}}$ is the length of the line source simulating the traffic emission, and $E_{\mathrm{s}}$ is the source strength) at the leeward wall of the $120 \mathrm{~cm}$-long street canyon with different traffic arrangements.

\section{Concentration and flow fields in the canyon with traffic}

\subsection{Concentration fields}

In this section we consider concentration distributions over the leeward walls of the upwind building of the long $(120 \mathrm{~cm})$ canyon with three different traffic arrangements: without traffic, with two-way traffic of $30 \mathrm{~km} / \mathrm{h}$, and with one-way traffic of the same density and velocity. The corresponding concentration patterns are presented in Fig. 4.

One may use these concentration distributions as indicators of the transport properties of the flow in the canyon under different traffic conditions. In the case without traffic, the maximum of concentration is observed around the central plane 
of the canyon. Due to ventilation through the outlets of the canyon, the concentration symmetrically decays towards the canyon edges. It is possible to see, however, that the noted symmetry is found also in the concentration pattern corresponding to the simulation case with two-way traffic. Such symmetry indicates that the two-way traffic does not generate any significant mean motion along the canyon. This conclusion will be later supported by results of the mean flow measurements. The lower concentration values in the two-way traffic case compared to the zero-traffic situation give an evidence of the additional diffusion of tracer due to the vehicle-induced turbulent motions. This diffusion is apparently invariant with respect to the sign of $y$.

When cars in both lanes move in the same direction, the situation is principally different. The concentration distribution in this case points to a strong piston effect leading to the skewed concentration field in the canyon. Hence, in the one-way traffic case, the car-induced mean air motion along the canyon plays the dominant role in the formation of the resulting concentration pattern. Presumably, the turbulent component of motion in this case also contributes to the dispersion of tracer, but its effect is clearly subordinate to the transport of gaseous admixture in the canyon by organised motion.

\subsection{Mean flow and turbulence fields}

Now we proceed to the wind tunnel measurements of the in-canyon flow for the cases of two-way and one-way traffic. The results of these measurements are presented in Fig. 5. The data are given as deviations of the measured flow characteristics in the long $(120 \mathrm{~cm})$ canyon with traffic from their counterparts in the long canyon without traffic. The employed scaling is the same as in Fig. 3.

Patterns of the longitudinal mean flow component $u$ (not shown) have not been found significantly altered by traffic. At the same time, the modification of the lateral velocity component $v$ essentially depends on the traffic arrangement. In the case of two-way traffic, the changes of $v$ are negligible (Fig. 5a, upper plot). This is consistent with our earlier observation of the symmetry of concentration distribution in the canyon with two-way traffic (Fig. 4). Only in the close vicinity of the leeward wall of the canyon a slight organised motion can be noted. This motion is associated with transport of momentum from the row of cars closest to this wall. The sign of transport is determined by the direction of the vortex-related motion in the lower portion of the canyon (see Fig. 3a).

When cars in both lanes move in the same direction (Fig. 5a, lower plot), they produce rather significant organised flow. Its mean velocity is non-uniformly distributed over the canyon cross-section. Due to the vortex-like mean circulation, the maximum of momentum in the lower portion of the canyon is shifted towards the upwind building which results in a spiral-type organised motion inside the canyon. This motion produces the aforementioned piston effect leading to the skewed concentration distribution along the canyon in the case with one-way traffic (see Fig. 4). 
(a) $\Delta v / u(4 H)$
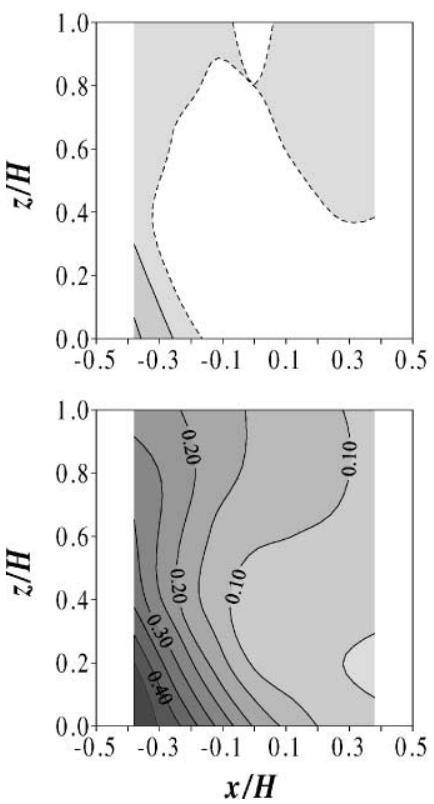

(b) $\Delta \sigma_{u} / u(4 H)$
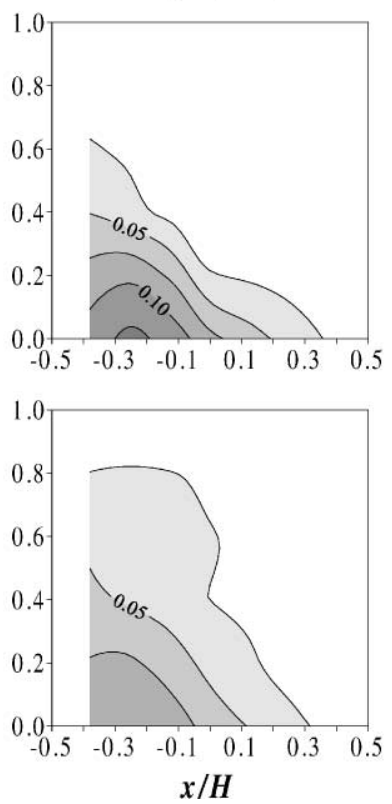

(c) $\Delta \sigma_{v} / u(4 H)$
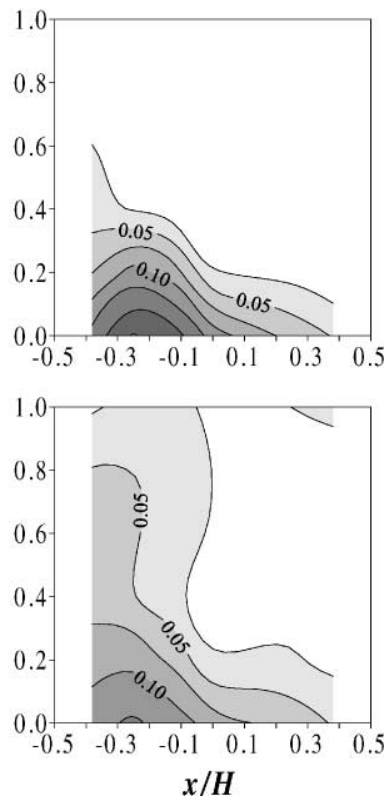

Fig. 5. Traffic-induced contributions to the normalised fields of the lateral (along-the-canyon) mean flow velocity component (a), and rms values of longitudinal (b) and lateral velocity (c) fluctuations inside the $120 \mathrm{~cm}$-long street canyon. The plots in the upper row refer to the case of two-way traffic and the lower row corresponds to the case of one-way traffic.

The spatial distribution of traffic-induced turbulent velocity fluctuations in the canyon is also asymmetric. Zones of large rms velocity values are persistently shifted towards the leeward wall of the canyon due to the near-floor advection associated with the in-canyon vortex. It is easy to notice that the magnitude of velocity fluctuations induced by traffic is larger in the two-way traffic case than in the oneway traffic case (compare upper and lower plots in Figs. 5a and b). Such turbulence enhancement is apparently a result of turbulence production in the separation zone between the lanes with cars moving in opposite directions. In this zone, the parcels of air pushed against each other by moving vehicles are mutually destroyed. This leads to the enhancement of the turbulent component of motion, but does not noticeably contribute to the mean transport along the canyon.

The situation in the one-way traffic case is different. With such traffic arrangement, one may expect mutual amplification of the momentum impulses of the same sign generated by vehicles moving in the parallel lanes. This results in the noticeable mean motion along the canyon, but does not generate turbulent motions as effectively as in the case with two-way traffic. Compared to real one-way traffic situations, the mean motion in the wind tunnel simulation is potentially exaggerated since the plates are constantly moving with the same velocity on both lanes whereas car velocities in 
nature are variable in space and time. This irregularity presumably leads to the attenuation of the generated mean motion.

\section{Summary and conclusions}

Organised and turbulent components of air motion in an urban street canyon oriented perpendicular to the external wind flow have been studied experimentally in a boundary-layer wind tunnel. A particular emphasis in the study has been laid on the interaction between the wind-related air motions and the component of motion induced by vehicles (cars) moving inside the canyon. The effects of vehicle-induced motions have been investigated for the cases of one-way and two-way traffic in the canyon.

For the case without traffic, the observed mean flow and turbulence profiles in the canyon have been analysed in comparison with data from corresponding field studies of flow structure in full-scale urban street canyons. Qualitative similarity has been found between the flow characteristics in the wind tunnel model and their atmospheric counterparts. The relatively large discrepancies between the wind tunnel and field measurement data have primarily been caused by the inappropriate scaling velocity $u(H)$, which had to be used due to data availability restrictions in the full-scale experiments. The insufficient information about the undisturbed-flow velocity, which could probably better serve for normalisation purposes, is a common problem for essentially all full-scale studies and thus deserves attention in further field experiments.

Data from all sources have presented evidence of the flow acceleration (in some cases, rather sharp) above the roof level. The shear production of turbulence in this acceleration region has apparently been responsible for relatively high rms values of horizontal velocity fluctuations above the canyon. Inside the canyon, the turbulence intensity has been found approximately uniformly distributed over the canyon crosssection. It has been suggested that turbulence is to a certain extent blocked inside the canyon due to shear sheltering in the flow-acceleration region above the roof level.

Values of the mean flow component $u$ across the canyon turned out to be practically unaffected by traffic, either one-way or two-way. In contrast to this, the along-the-canyon mean flow component $v$ has displayed strong dependence on the traffic arrangement in the canyon. With two-way traffic, the traffic-induced component of the mean air motion along the canyon has been very small and restricted to the flow region in the vicinity of the leeward wall. At the same time, the turbulence intensity inside the canyon with two-way traffic has been markedly larger than in the case of no traffic and one-way traffic. The case of one-way traffic has been characterised by the traffic-induced organised air motion along the canyon leading to a pronounced piston effect.

Relations between flow and concentration patterns in the canyon with different traffic arrangements have been investigated. In the case of quasi-isotropic turbulent transport corresponding to the two-way traffic situation, the concentration distribution in the canyon has been found to be approximately symmetric with 
respect to the central transversal plane of the canyon. Mean transport of tracer gas by vehicle-induced air motions has been negligibly small in this case. With the organised motion induced by one-way traffic, the concentration distribution along the canyon has displayed strong lateral skewness, with concentration values increasing in the direction of traffic-induced air current.

\section{Acknowledgements}

The authors gratefully acknowledge financial support provided for the presented study by the Training and Mobility of Researchers Programme of the European Commission within the TRAPOS Project, and by the Air Pollution Prevention Measures Programme (PEF) funded by the Land of Baden-Württemberg (Germany) and the European Commission.

\section{References}

[1] G.T. Johnson, L.J. Hunter, A numerical study of dispersion of passive scalars in city canyons, Boundary-Layer Meteorol. 75 (1995) 235-262.

[2] J.-F. Sini, S. Anquetin, P.G. Mestayer, Pollutant dispersion and thermal effects in urban street canyons, Atmos. Environ. 30 (1996) 2659-2677.

[3] B.M. Leitl, R.N. Meroney, Car exhaust dispersion in a street canyon. Numerical critique of a wind tunnel experiment, J. Wind Eng. Ind. Aerodyn. 67\&68 (1997) 293-304.

[4] W.F. Dabberdt, W.G. Hoydysh, Street canyon dispersion: sensitivity to block shape and entrainment, Atmos. Environ. 25 A (1991) 1143-1153.

[5] S. Rafailidis, Influence of building areal density and roof shape on the wind characteristics above a town, Boundary-Layer Meteorol. 85 (1997) 255-271.

[6] O. Hertel, R. Berkowicz, Modelling pollution from traffic in a street canyon. Evaluation of data and model development, DMU Luft A-129, 1989, 77pp.

[7] R. Stern, R.J. Yamartino, Development and initial application of the micro-calgrid photochemical model for high-resolution studies of urban environments, Proceedings of the 23rd NATO/CCMS ITM on Air Pollution Modelling and its Applications, Varna, Bulgaria, 1998.

[8] R.E. Eskeridge, J.C.R. Hunt, Highway modeling. Part I: prediction of velocity and turbulence fields in the wake of vehicles, J. Appl. Meteorol. 18 (1979) 387-400.

[9] D. Delaunay, N. Houseaux, Turbulence et quantite de mouvement induites par les vehicules a proximite d'une voie urbaine: mesures in situ, Centre Scientifique et Technique du Batiment (CSTB), Nantes, Report EN-AEC 97, 1997, 68pp.

[10] P. Kastner-Klein, R. Berkowicz, E.J. Plate, Modelling of vehicle induced turbulence in air pollution studies for streets, Int. J. Environ. Pollut. 14 (2000) 496-507.

[11] P. Kastner-Klein, E. Fedorovich, E.J. Plate, Gaseous pollutant dispersion around urban-canopy elements: wind tunnel case studies, Int. J. Environ. Pollut. 8 (1997) 727-737.

[12] P. Kastner-Klein, Experimentelle Untersuchung der strömungsmechanischen Transport-vorgänge in Straßenschluchten, Dissertation an der Fakultät für Bauingeneur- und Vermessungswesen der Universität Karlsruhe (TH), 1999.

[13] W. Brilon, H.J. Niemann, E. Romberg, Windkanaluntersuchungen zur Ausbreitung von Abgasen an Autobahnen, Straßenverkehrstechnik 31 (1987) 122-133.

[14] M.W. Rotach, Profiles of turbulence statistics in and above an urban street canyon, Atmos. Environ. 29 (1995) 1473-1486. 
[15] P. Louka, Measurements of airflow in an urban environment, Ph.D. Thesis, University of Reading, UK, 1998.

[16] M.J. Brown, R.E. Lawson, D.S. Descroix, R.L. Lee, Mean flow and turbulence measurements around a 2-D array of buildings in a wind tunnel. Proceedings of the 11th Conference on Appl. Air Poll. Met., Long Beach, CA, USA, 2000.

[17] E. Fedorovich, R. Kaiser, Wind tunnel model study of turbulence regime in the atmospheric convective boundary layer, in: E.J. Plate et al. (Eds.), Buoyant Convection in Geophysical Flows, Kluwer, Dordrecht, 1998, pp. 327-370.

[18] J.C.R. Hunt, Eddy dynamics and kinematics of convective turbulence, in: E.J. Plate et al. (Eds.), Buoyant Convection in Geophysical Flows, Kluwer, Dordrecht, 1998, pp. 41-82. 